\title{
Brauchen wir bei der akuten Exazerbation der COPD Antibiotika?
}

\author{
Der Stellenwert von Antibiotika bei \\ akuter Exazerbation einer COPD (AE- \\ COPD) wird kontrovers diskutiert, \\ und ihre Effizienz zusätzlich zu sys- \\ temischen Steroiden ist unbekannt. \\ Daher wurde eine randomisierte, \\ placebokontrollierte Studie mit \\ dem Ziel durchgeführt, die Effekte \\ von Doxycyclin zusätzlich zu sys- \\ temischen Steroiden bei hospitali- \\ sierten Patienten mit AE-COPD zu \\ erfassen.
}

- AE-COPD wurde als Zunahme von Luftnot und Sputumvolumen mit oder ohne vermehrte Sputumpurulenz definiert. Ausgewertet wurden Exazerbationen bei 233 Patienten. Die Patienten erhielten 200 mg Doxycyclin oder Placebo für sieben Tage zusätzlich zu systemischen Steroiden.

Das klinische und mikrobiologische Ansprechen, die Zeit bis zum Therapieversagen, die Lungenfunktion und Symptome sowie CRP wurden ausgewertet. Der klinische Erfolg an Tag 30 (primärer Endpunkt) war gleich so-

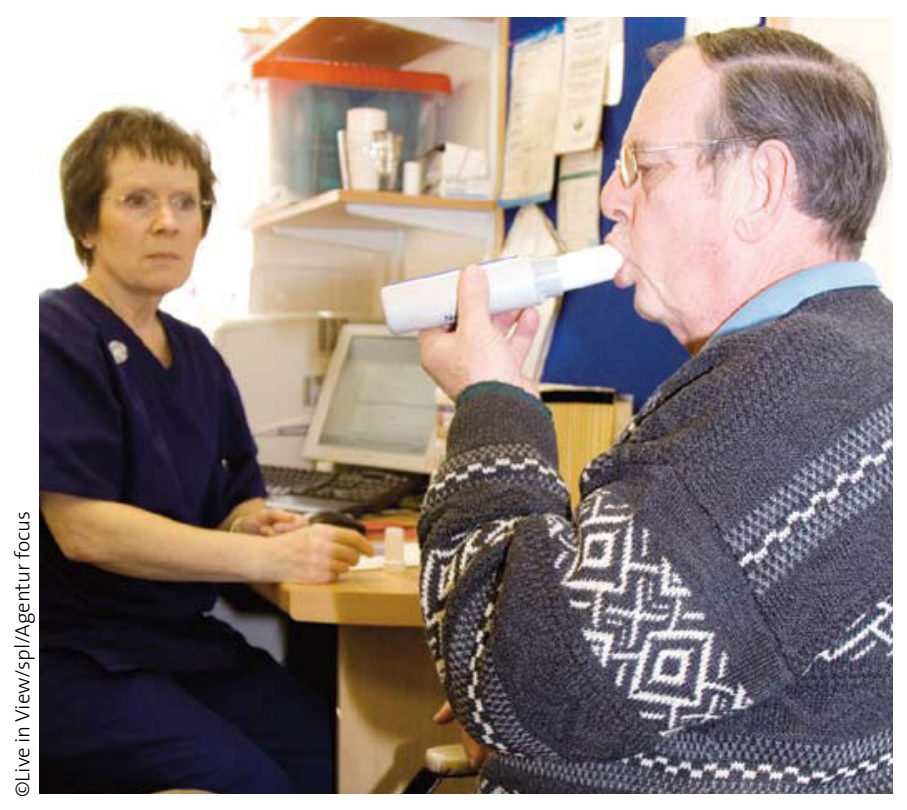

4 Seine COPD ist akut schlimmer geworden. Welche Therapie braucht er jetzt? wohl in der Intent-to-treat-Gruppe (OR 1,3; 95\%-KI 0,8-2,0) als auch in der Per-Protocol-Gruppe.

Obwohl die Therapie mit Doxycyclin in Bezug auf den primären Endpunkt klinischer Erfolg an Tag 30 äquivalent zu Placebo war, zeigte sich eine
Überlegenheit beim klinischen Erfolg und bei der klinischen Heilung an Tag 10, dem mikrobiologischen Ansprechen, dem Gebrauch zusätzlicher Antibiotika und der Reduktion von Symptomen.

\section{Kommentar}

Der Stellenwert einer antibiotischen Therapie bei der AE-COPD wird seit Langem sehr kontrovers diskutiert. Nichtsdestotrotz werden gerade bei dieser Indikation mit die meisten Antibiotika verschrieben. Die Datenlage ist hierbei extrem spärlich, was in krassem Gegensatz zur Häufigkeit und Ausprägung von AECOPD steht. Vor diesem Hintergrund stellt die hier vorgestellte Studie sicherlich einen Fortschritt dar. Alle Patienten erhielten intravenös Steroide: initial $60 \mathrm{mg}$ Prednisolon. Weiterhin wurden sie vier bis sechsmal pro Tag mit Bronchodilatatoren vernebelt und Physiotherapie durchgeführt. Die vorbestehende COPDMedikation wurde unverändert fortgeführt außer kurzwirksame Bronchodilatatoren. Der primäre Endpunkt wurde nicht erreicht, allerdings fanden sich Vorteile für Doxycyclin für weitere Endpunkte (s.o.).

Die Autoren diskutieren, dass das Fehlen eines signifikanten Effektes von Doxycyclin unter anderem dadurch zu erklären sei, dass Fieber und Röntgenbilder, die einen Verdacht auf Pneumo- nie zeigten, Ausschlusskriterien waren und dass es sich um AECOPD handelte, die eine Hospitalisation bedingten, also einen Schweregrad hatten, der frühe Rückfälle unter Therapie begünstige. Weiterhin stellt sich natürlich die Frage, ob Doxycyclin das geeignete Antibiotikum für diese Patientengruppe darstellte. Für eine belastbare Subgruppenanalyse war die Studie eindeutig zu klein.

Zusammengefasst ist der Weg, Antibiotika zusätzlich zu einer sicheren Standardtherapie der AE-COPD zu untersuchen, sicher richtig. Diese Studie hat jedoch so viele Limitationen, dass die Frage nach dem Stellenwert von Antibiotika bei der AE-COPD nicht beantwortet werden kann. Es wird weiterhin dringend eine ausreichend große Multicenterstudie mit einem sicher wirksamen Antibiotikum benötigt.

G. ROHDE :

- J. M. Daniels et al.

Antibiotics in addition to systemic corticosteroids for acute exacerbations of COPD: AJRCCM 181 (2010) 150-157 